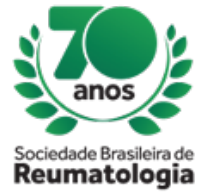

\title{
RITUXIMAB IN POLYARTERITIS CYCLOPHOSPHAMIDE INTOLERANT NODOSE: CASE REPORT
}

Adah Sophia Rodrigues Vieira (Universidade de Fortaleza, Fortaleza, CE, Brasil), Guilherme Lopes Pereira (Universidade de Fortaleza, Fortaleza, CE, Brasil), Antônio José da Silva Neto (Hospital Geral de Fortaleza, Fortaleza, CE, Brasil), Aline de Castro Caracas (Universidade de Fortaleza, Fortaleza, CE, Brasil), Rodrigo Barbosa De Azevedo (Hospital Geral Dr. César Cals, Fortaleza, CE, Brasil), Rejane Maria Rodrigues de Abreu Vieira (Hospital Geral de Fortaleza, Universidade de Fortaleza e Universidade Estadual do Ceará, Fortaleza, CE, Brasil)

\section{BACKGROUND}

Polyarteritis nodosa (PAN) is a rare vasculitis, reaching 6 per 100,000 individuals, affecting mainly men in the fifth decade of life. Despite the systemic involvement, presenting nonspecific symptoms such as fever, asthenia, and arthralgia, most of the symptoms are of cutaneous origins, such as livedo reticularis, Raynaud's phenomenon, ulcers and ischemic alterations of the fingertips and calcaneus. If not diagnosed, it can be fatal due to renal, cardiovascular, and neurological repercussions. The use of glucocorticoids and cyclophosphamide in the remission of this disease changed the prognosis and survival of patients. Unfortunately, some cases do not respond to or tolerate this medication, and failure to treat it can be fatal in most cases. With a higher definition of the role of $B$ lymphocytes in the pathogenesis of these diseases, the use of rituximab, an anti-CD20 monoclonal antibody, appears as a therapeutic option. The aim is to report the case of a young woman with PAN, treated with anti-CD20 monoclonal antibody.

\section{CASE REPORT}

A 42-year-old woman, in 2015, initiated symptoms of paresthesia in the lower limbs and afternoon fever. Slow and progressive evolution, with the appearance of livedo reticularis, Raynaud's phenomenon, areas of necrosis in the 4th left pododactyl and ulcer in the 5th right chirodactyl, being admitted for investigation. Due to the chronic and intense ischemic pain, the patient used exacerbated potent analgesics and morphine. FAN, rheumatoid factor, P and C-ANCA tests, and research for thrombophilia were negative. Serology for hepatitis $B$ and $C$ was also negative. An electroneuromyography of the upper and lower limbs showed multiple confluent mononeuritis. A skin biopsy suggestive of vasculitis. She was treated with corticosteroids, with low adherence to the treatment because she presented depressive condition, besides Cyclophosphamide pulses. However, the patient presented severe infectious complications, making continuity impossible. Then, it was decided to use the rituximab, the patient performed two biweekly doses of $1 \mathrm{~g}$ of medication and showed marked improvement in ischemic lesions. Six-monthly maintenance infusions were scheduled.

\section{CONCLUSION}

Because polyarteritis nodosa is a rare and debilitating disease, it requires immediate diagnostic and therapeutic efficacy. Rituximab has proven to be a promising option for patients with PAN, with some intolerance or refractoriness to standard therapies. 